\title{
Unique solutions for a new coupled system of fractional differential equations
}

\section{Chengbo Zhai* (D) and Ruiting Jiang}

"Correspondence:
cbzhai@sxu.edu.cn
School of Mathematical Sciences,
Shanxi University, Taiyuan, Shanxi
030006, P.R. China

*Correspondence:

School of Mathematical Sciences,

030006, P.R. China

\begin{abstract} with integral boundary conditions

$$
\begin{cases}D^{\alpha} u(t)+f(t, v(t))=a, & 0<t<1, \\ D^{\beta} v(t)+g(t, u(t))=b, & 0<t<1, \\ u(0)=0, & u(1)=\int_{0}^{1} \phi(t) u(t) d t, \\ v(0)=0, & v(1)=\int_{0}^{1} \psi(t) v(t) d t,\end{cases}
$$
\end{abstract}

In this article, we discuss a new coupled system of fractional differential equations

where $1<\alpha, \beta \leq 2, f, g \in C([0,1] \times(-\infty,+\infty),(-\infty,+\infty)), \phi, \psi \in L^{1}[0,1], a, b$ are constants and $D$ denotes the usual Riemann-Liouville fractional derivative. Based upon a fixed point theorem of increasing $\varphi$-(h,e)-concave operators, we establish the existence and uniqueness of solutions for the new coupled system dependent on two constants. And then the obtained result is well demonstrated with the aid of an interesting example.

MSC: 34A08; 26A33; 34B18; 34B27

Keywords: existence and uniqueness; coupled system of fractional differential equations; integral boundary condition; $\varphi$-(h,e)-concave operator

\section{Introduction}

In this article, we study a new coupled system of fractional differential equations and consider the existence and uniqueness of solutions for the system with integral boundary conditions. Namely, we discuss the following problem:

$$
\left\{\begin{array}{l}
D^{\alpha} u(t)+f(t, v(t))=a, \quad 0<t<1, \\
D^{\beta} v(t)+g(t, u(t))=b, \quad 0<t<1, \\
u(0)=0, \quad u(1)=\int_{0}^{1} \phi(t) u(t) d t, \\
v(0)=0, \quad v(1)=\int_{0}^{1} \psi(t) v(t) d t,
\end{array}\right.
$$

where $1<\alpha, \beta \leq 2, f, g \in C([0,1] \times(-\infty,+\infty),(-\infty,+\infty)), \phi, \psi \in L^{1}[0,1], a, b$ are constants and $D$ denotes the usual Riemann-Liouville fractional derivative (see [1, 2]). A pair of functions $(u, v) \in C([0,1]) \times C([0,1])$ is called a solution of system $(1.1)$ if it satisfies $(1.1)$.

(c) The Author(s) 2018. This article is distributed under the terms of the Creative Commons Attribution 4.0 International License (http://creativecommons.org/licenses/by/4.0/), which permits unrestricted use, distribution, and reproduction in any medium, provided you give appropriate credit to the original author(s) and the source, provide a link to the Creative Commons license, and indicate if changes were made. 
We will consider system (1.1) under the case $a>0, b>0$. When $a=b=0$, we can study problem (1.1) by using the usual methods and give some results about the existence and uniqueness of positive solutions (see [3]). However, when $a, b>0$, we cannot get similar results by using the previous methods. So system (1.1) is new, and we need to seek new methods to discuss problem (1.1). Fortunately, we can use a recent fixed point theorem for $\varphi$-(h,e)-concave operators to resolve problem (1.1). First, we list the following hypotheses on the functions $\phi(t), \psi(t)$ :

(Q) $\phi, \psi:[0,1] \rightarrow[0,+\infty)$ with $\phi, \psi \in L^{1}[0,1]$ and

$$
\begin{aligned}
& \sigma_{1}:=\int_{0}^{1} \phi(t) t^{\alpha-1} d t, \quad \sigma_{2}:=\int_{0}^{1} \psi(t) t^{\beta-1} d t \in(0,1) \\
& \sigma_{3}:=\int_{0}^{1} t^{\alpha-1}(1-t) \phi(t) d t, \quad \sigma_{4}:=\int_{0}^{1} t^{\beta-1}(1-t) \psi(t) d t>0 .
\end{aligned}
$$

A lot of boundary value problems of coupled systems involving fractional differential equations have been investigated extensively, see the works [4-25] and the references therein. Different boundary conditions of coupled systems can be found in the discussions of some problems such as Sturm-Liouville problems and some reaction-diffusion equations (see $[26,27])$, and they have some applications in many fields such as mathematical biology (see $[28,29]$ ), natural sciences and engineering; for example, we can see beam deformation and steady-state heat flow [30,31] and heat equations [14, 32, 33]. So nonlinear coupled systems subject to different boundary conditions have been paid much attention to, and the existence or multiplicity of solutions for the systems has been given in literature, see [4-14, 16-25] for example. The usual methods used are Schauder's fixed point theorem, Banach's fixed point theorem, Guo-Krasnosel'skii's fixed point theorem on cone, nonlinear differentiation of Leray-Schauder type and so on. Recently, several papers $[4,8-10]$ considered some new coupled systems of fractional differential equations and obtained some new results about the existence and uniqueness of solutions by using general methods.

From literature, no papers have considered system (1.1). Inspired by the works of coupled systems and recent papers [16,34], we study the coupled system (1.1) and give the existence and uniqueness of solutions. By using a fixed point theorem of increasing $\varphi$ $(h, e)$-concave operators, we establish the existence and uniqueness of solutions for the coupled system dependent on two constants. Our result shows that the unique solution exists in a product set and can be approximated by making an iterative sequence for any initial point in the product set. So our result is an extension and improvement of the previous works.

\section{Preliminaries}

Lemma 2.1 (see [11]) Let $\int_{0}^{1} \phi(t) t^{\alpha-1} d t \neq 1$ and $\sigma \in C[0,1]$. Then the problem

$$
\left\{\begin{array}{l}
D^{\alpha} u(t)+\sigma(t)=0, \quad 0<t<1, \\
u(0)=0, \quad u(1)=\int_{0}^{1} \phi(t) u(t) d t
\end{array}\right.
$$


has the unique solution $u(t)=\int_{0}^{1} G_{1 \alpha}(t, s) \sigma(s) d s$, where $G_{1 \alpha}(t, s)=G_{2 \alpha}(t, s)+G_{3 \alpha}(t, s)$ with

$$
\begin{aligned}
& G_{2 \alpha}(t, s)= \begin{cases}\frac{t^{\alpha-1}(1-s)^{\alpha-1}-(t-s)^{\alpha-1}}{\Gamma(\alpha)}, & 0 \leq s \leq t \leq 1, \\
\frac{t^{\alpha-1}(1-s)^{\alpha-1}}{\Gamma(\alpha)}, & 0 \leq t \leq s \leq 1,\end{cases} \\
& G_{3 \alpha}(t, s)=\frac{t^{\alpha-1}}{1-\int_{0}^{1} \phi(t) t^{\alpha-1} d t} \int_{0}^{1} \phi(t) G_{2 \alpha}(t, s) d t .
\end{aligned}
$$

The function $G(t, s)=\left(G_{1 \alpha}(t, s), G_{1 \beta}(t, s)\right)$ is the Green's function of problem (1.1).

Lemma 2.2 (see [11]) Let $\int_{0}^{1} \phi(t) t^{\alpha-1} d t \in[0,1)$. Then $G_{1 \alpha}(t, s) \geq 0$ is continuous for all $t, s \in[0,1]$, and $G_{1 \alpha}(t, s)>0$ for all $t, s \in(0,1)$.

Lemma 2.3 (see [3]) The function $G_{2 \alpha}(t, s)$ satisfies

$$
\frac{\alpha-1}{\Gamma(\alpha)} t^{\alpha-1}(1-t)(1-s)^{\alpha-1} s \leq G_{2 \alpha}(t, s) \leq \frac{t^{\alpha-1}(1-s)^{\alpha-1}}{\Gamma(\alpha)}, \quad t, s \in[0,1]
$$

Lemma 2.4 (see [3]) Suppose $\alpha, \beta \in(1,2]$ and $(Q)$ is satisfied. Then the functions $G_{1 \alpha}(t, s)$, $G_{1 \beta}(t, s)$ satisfy

$$
\begin{aligned}
& \frac{(\alpha-1) \sigma_{3} s(1-s)^{\alpha-1} t^{\alpha-1}}{\left(1-\sigma_{1}\right) \Gamma(\alpha)} \leq G_{1 \alpha} \leq \frac{(1-s)^{\alpha-1} t^{\alpha-1}}{\Gamma(\alpha)\left(1-\sigma_{1}\right)}, \quad t, s \in[0,1] \\
& \frac{(\beta-1) \sigma_{4} s(1-s)^{\beta-1} t^{\beta-1}}{\left(1-\sigma_{2}\right) \Gamma(\beta)} \leq G_{1 \beta} \leq \frac{(1-s)^{\beta-1} t^{\beta-1}}{\Gamma(\beta)\left(1-\sigma_{2}\right)}, \quad t, s \in[0,1] .
\end{aligned}
$$

Next we present a fixed point theorem which can be easily used to study some systems of differential equations.

Suppose $(E,\|\cdot\|)$ is a real Banach space and it is partially ordered by a cone $P \subset E$. For any $x, y \in E, x \sim y$ denotes that there are $\mu>0$ and $v>0$ such that $\mu x \leq y \leq v x$. Take $h>\theta$ (i.e., $h \geq \theta$ and $h \neq \theta$ ), we consider the set $P_{h}=\{x \in E \mid x \sim h\}$. Clearly, $P_{h} \subset P$. Take another element $e \in P$ with $\theta \leq e \leq h$, we define $P_{h, e}=\left\{x \in E \mid x+e \in P_{h}\right\}$.

Definition 2.1 (see [34]) Assume that $A: P_{h, e} \rightarrow E$ is an operator which satisfies: for any $x \in P_{h, e}$ and $\lambda \in(0,1)$, there exists $\varphi(\lambda)>\lambda$ such that $A(\lambda x+(\lambda-1) e) \geq \varphi(\lambda) A x+(\varphi(\lambda)-1) e$. Then we call $A$ a $\varphi$ - $(h, e)$-concave operator.

Lemma 2.5 (see [34]) Suppose that $P$ is normal and $A$ is an increasing $\varphi-(h, e)$-concave operator satisfying $A h \in P_{h, e}$. Then $A$ has a unique fixed point $x^{*}$ in $P_{h, e}$. In addition, for any $w_{0} \in P_{h, e}$, constructing the sequence $w_{n}=A w_{n-1}, n=1,2, \ldots$, then $\left\|w_{n}-x^{*}\right\| \rightarrow 0$ as $n \rightarrow \infty$.

Given $h_{1}, h_{2} \in P$ with $h_{1}, h_{2} \neq \theta$. Let $h=\left(h_{1}, h_{2}\right)$, then $h \in \bar{P}:=P \times P$. Take $\theta \leq e_{1} \leq h_{1}$, $\theta \leq e_{2} \leq h_{2}$, and let $\bar{\theta}=(\theta, \theta), e=\left(e_{1}, e_{2}\right)$. Then $\bar{\theta}=(\theta, \theta) \leq\left(e_{1}, e_{2}\right) \leq\left(h_{1}, h_{2}\right)=h$. That is, $\bar{\theta} \leq e \leq h$. If $P$ is normal, then $\bar{P}=P \times P$ is normal (see [35]).

Lemma 2.6 (see [3]) $\overline{P_{h}}=P_{h_{1}} \times P_{h_{2}}$. 
Lemma 2.7 $\bar{P}_{h, e}=P_{h_{1}, e_{1}} \times P_{h_{2}, e_{2}}$.

Proof By Lemma 2.6, we obtain

$$
\begin{aligned}
\bar{P}_{h, e} & =\left\{(x, y) \in E \times E \mid(x, y)+e \in \bar{P}_{h}\right\} \\
& =\left\{(x, y) \in E \times E \mid\left(x+e_{1}, y+e_{2}\right) \in P_{h_{1}} \times P_{h_{2}}\right\} \\
& =\left\{(x, y) \in E \times E \mid x+e_{1} \in P_{h_{1}}, y+e_{2} \in P_{h_{2}}\right\}=\left\{(x, y) \in E \times E \mid x \in P_{h_{1}, e_{1}}, y \in P_{h_{2}, e_{2}}\right\} \\
& =P_{h_{1}, e_{1}} \times P_{h_{2}, e_{2}} .
\end{aligned}
$$

\section{Existence and uniqueness of solutions}

In this section, let $E=\{u \mid u \in C[0,1]\}$ and the norm of $E$ is $\|u\|=\max _{t \in[0,1]}|u(t)|$. We will consider (1.1) in $E \times E$. For $(u, v) \in E \times E$, let $\|(u, v)\|=\max \{\|u\|,\|v\|\}$. It is clear that $(E \times$ $E,\|(u, v)\|)$ is a Banach space. Let $\bar{P}=\{(u, v) \in E \times E \mid u(t) \geq 0, v(t) \geq 0\}, P=\{u \in E \mid u(t) \geq$ $0, t \in[0,1]\}$, then the cone $\bar{P} \subset E \times E$ and $\bar{P}=P \times P$ is normal, and the space $E \times E$ has a partial order: $\left(u_{1}, v_{1}\right) \leq\left(u_{2}, v_{2}\right) \Leftrightarrow u_{1}(t) \leq u_{2}(t), v_{1}(t) \leq v_{2}(t), t \in[0,1]$. By Lemma 2.1 and the result of [21], we can easily get the following result.

Lemma 3.1 Suppose that $(Q)$ is satisfied and $f(t, x), g(t, x)$ are continuous, then $(u, v) \in$ $E \times E$ is a solution of (1.1) if and only if $(u, v) \in E \times E$ is a solution of the following equations:

$$
\left\{\begin{array}{l}
u(t)=\int_{0}^{1} G_{1 \alpha}(t, s) f(s, v(s)) d s-a \int_{0}^{1} G_{1 \alpha}(t, s) d s, \\
v(t)=\int_{0}^{1} G_{1 \beta}(t, s) g(s, u(s)) d s-b \int_{0}^{1} G_{1 \beta}(t, s) d s .
\end{array}\right.
$$

For $(u, v) \in E \times E$, we define three operators $A_{1}, A_{2}$ and $T$ by

$$
\begin{aligned}
& A_{1} u(t)=\int_{0}^{1} G_{1 \alpha}(t, s) f(s, v(s)) d s-a \int_{0}^{1} G_{1 \alpha}(t, s) d s, \\
& A_{2} v(t)=\int_{0}^{1} G_{1 \beta}(t, s) g(s, u(s)) d s-b \int_{0}^{1} G_{1 \beta}(t, s) d s,
\end{aligned}
$$

and $T(u, v)(t)=\left(A_{1} u(t), A_{2} v(t)\right)$. Then $A_{1}, A_{2}: E \rightarrow E$ and $T: E \times E \rightarrow E \times E$. From Lemma 3.1, $(u, v)$ is the solution of system (1.1) if and only if $(u, v)$ is the fixed point of operator $T$. Let

$$
\begin{aligned}
& e_{1}(t)=a \int_{0}^{1} G_{1 \alpha}(t, s) d s, \quad e_{2}(t)=b \int_{0}^{1} G_{1 \beta}(t, s) d s, \\
& h_{1}(t)=M_{1} t^{\alpha-1}, \quad h_{2}(t)=M_{2} t^{\beta-1},
\end{aligned}
$$

where $M_{1} \geq \frac{a}{\Gamma(\alpha+1)\left(1-\sigma_{1}\right)}, M_{2} \geq \frac{b}{\Gamma(\beta+1)\left(1-\sigma_{2}\right)}$.

Theorem 3.1 Let $1<\alpha, \beta \leq 2, a>0, b>0$ and $e_{1}, e_{2}, h_{1}, h_{2}$ be given as in (3.1). Assume that $f, g \in C([0,1] \times(-\infty,+\infty),(-\infty,+\infty))$ and $(Q)$ holds. Moreover,

$\left(H_{1}\right) f:[0,1] \times\left[-e_{2}^{*},+\infty\right) \rightarrow(-\infty,+\infty)$ is increasing with respect to the second variable, where $e_{2}^{*}=\max \left\{e_{2}(t): t \in[0,1]\right\} ; g:[0,1] \times\left[-e_{1}^{*},+\infty\right) \rightarrow(-\infty,+\infty)$ is increasing with respect to the second variable, where $e_{1}^{*}=\max \left\{e_{1}(t): t \in[0,1]\right\}$; 
$\left(H_{2}\right)$ for $\lambda \in(0,1)$, there exists $\varphi(\lambda)>\lambda$ such that

$$
\begin{array}{ll}
f(t, \lambda x+(\lambda-1) y) \geq \varphi(\lambda) f(t, x), & t \in[0,1], x \in(-\infty,+\infty), y \in\left[0, e_{2}^{*}\right] \\
g(t, \lambda x+(\lambda-1) y) \geq \varphi(\lambda) g(t, x), & t \in[0,1], x \in(-\infty,+\infty), y \in\left[0, e_{1}^{*}\right]
\end{array}
$$

$\left(H_{3}\right) f(t, 0) \geq 0, g(t, 0) \geq 0$ with $f(t, 0) \not \equiv 0, g(t, 0) \not \equiv 0$ for $t \in[0,1]$.

Then:

(1) system (1.1) has a unique solution $\left(u^{*}, v^{*}\right)$ in $\bar{P}_{h, e}$, where

$$
e(t)=\left(e_{1}(t), e_{2}(t)\right), \quad h(t)=\left(h_{1}(t), h_{2}(t)\right), \quad t \in[0,1]
$$

(2) taking any point $\left(u_{0}, v_{0}\right) \in \bar{P}_{h, e}$, construct the following sequences:

$$
\begin{gathered}
u_{n+1}(t)=\int_{0}^{1} G_{1 \alpha}(t, s) f\left(s, v_{n}(s)\right) d s-a \int_{0}^{1} G_{1 \alpha}(t, s) d s, \\
v_{n+1}(t)=\int_{0}^{1} G_{1 \beta}(t, s) g\left(s, u_{n}(s)\right) d s-b \int_{0}^{1} G_{1 \beta}(t, s) d s, \\
n=0,1,2, \ldots, \text { we have } u_{n+1}(t) \rightarrow u^{*}(t), v_{n+1}(t) \rightarrow v^{*}(t) \text { as } n \rightarrow \infty .
\end{gathered}
$$

Proof By Lemma 2.2, for $t \in[0,1]$,

$$
e_{1}(t)=a \int_{0}^{1} G_{1 \alpha}(t, s) d s \geq 0, \quad e_{2}(t)=b \int_{0}^{1} G_{1 \beta}(t, s) d s \geq 0
$$

From Lemma 2.4, for $t \in[0,1]$,

$$
\begin{aligned}
e_{1}(t) & =a \int_{0}^{1} G_{1 \alpha}(t, s) d s \leq a \int_{0}^{1} \frac{(1-s)^{\alpha-1} t^{\alpha-1}}{\Gamma(\alpha)\left(1-\sigma_{1}\right)}=\frac{a t^{\alpha-1}}{\Gamma(\alpha)\left(1-\sigma_{1}\right)} \int_{0}^{1}(1-s)^{\alpha-1} d s \\
& =\frac{a t^{\alpha-1}}{\alpha \Gamma(\alpha)\left(1-\sigma_{1}\right)}=\frac{a}{\Gamma(\alpha+1)\left(1-\sigma_{1}\right)} t^{\alpha-1} \leq M_{1} t^{\alpha-1}=h_{1}(t) ; \\
e_{2}(t) & =b \int_{0}^{1} G_{1 \beta}(t, s) d s \leq b \int_{0}^{1} \frac{(1-s)^{\beta-1} t^{\beta-1}}{\Gamma(\beta)\left(1-\sigma_{2}\right)}=\frac{b t^{\beta-1}}{\Gamma(\beta)\left(1-\sigma_{2}\right)} \int_{0}^{1}(1-s)^{\beta-1} d s \\
& =\frac{b t^{\beta-1}}{\beta \Gamma(\beta)\left(1-\sigma_{2}\right)}=\frac{b}{\Gamma(\beta+1)\left(1-\sigma_{2}\right)} t^{\beta-1} \leq M_{2} t^{\beta-1}=h_{2}(t) .
\end{aligned}
$$

That is, $0 \leq e_{1} \leq h_{1}, 0 \leq e_{2} \leq h_{2}$.

In the following, we prove that $T: \bar{P}_{h, e} \rightarrow E \times E$ is a $\varphi$ - $(h, e)$-concave operator. For $(u, v) \in$ $\bar{P}_{h, e}, \lambda \in(0,1)$, we obtain

$$
\begin{aligned}
T & (\lambda(u, v)+(\lambda-1) e)(t) \\
& =T\left(\lambda(u, v)+(\lambda-1)\left(e_{1}, e_{2}\right)\right)(t) \\
& =T\left(\lambda u+(\lambda-1) e_{1}, \lambda v+(\lambda-1) e_{2}\right)(t) \\
& =\left(A_{1}\left(\lambda u+(\lambda-1) e_{1}\right), A_{2}\left(\lambda v+(\lambda-1) e_{2}\right)\right)(t) .
\end{aligned}
$$


We discuss $A_{1}\left(\lambda u+(\lambda-1) e_{1}\right)(t)$ and $A_{2}\left(\lambda v+(\lambda-1) e_{2}\right)(t)$ respectively. From $\left(H_{2}\right)$,

$$
\begin{aligned}
A_{1} & \left(\lambda u+(\lambda-1) e_{1}\right)(t) \\
& =\int_{0}^{1} G_{1 \alpha}(t, s) f\left(s, \lambda v(s)+(\lambda-1) e_{2}(s)\right) d s-e_{1}(t) \\
& \geq \varphi(\lambda) \int_{0}^{1} G_{1 \alpha}(t, s) f(s, v(s)) d s-e_{1}(t) \\
& =\varphi(\lambda)\left[\int_{0}^{1} G_{1 \alpha}(t, s) f(s, v(s)) d s-e_{1}(t)\right]+[\varphi(\lambda)-1] e_{1}(t) \\
& =\varphi(\lambda) A_{1} u(t)+[\varphi(\lambda)-1] e_{1}(t), \\
A_{2} & \left(\lambda v+(\lambda-1) e_{2}\right)(t) \\
& =\int_{0}^{1} G_{1 \beta}(t, s) g\left(s, \lambda u(s)+(\lambda-1) e_{1}(s)\right) d s-e_{2}(t) \\
& \geq \varphi(\lambda) \int_{0}^{1} G_{1 \beta}(t, s) g(s, u(s)) d s-e_{2}(t) \\
& =\varphi(\lambda)\left[\int_{0}^{1} G_{1 \beta}(t, s) g(s, u(s)) d s-e_{2}(t)\right]+[\varphi(\lambda)-1] e_{2}(t) \\
& =\varphi(\lambda) A_{2} v(t)+[\varphi(\lambda)-1] e_{2}(t) .
\end{aligned}
$$

So we have

$$
\begin{aligned}
T & (\lambda(u, v)+(\lambda-1) e)(t) \\
& \geq\left(\varphi(\lambda) A_{1} u(t)+[\varphi(\lambda)-1] e_{1}(t), \varphi(\lambda) A_{2} v(t)+[\varphi(\lambda)-1] e_{2}(t)\right) \\
& =\left(\varphi(\lambda) A_{1} u(t), \varphi(\lambda) A_{2} v(t)\right)+\left((\varphi(\lambda)-1) e_{1}(t),(\varphi(\lambda)-1) e_{2}(t)\right) \\
& =\varphi(\lambda)\left(A_{1} u(t), A_{2} v(t)\right)+(\varphi(\lambda)-1)\left(e_{1}(t), e_{2}(t)\right) \\
& =\varphi(\lambda) T(u, v)(t)+(\varphi(\lambda)-1) e(t) .
\end{aligned}
$$

That is,

$$
\begin{aligned}
& T(\lambda(u, v)+(\lambda-1) e) \\
& \quad \geq \varphi(\lambda) T(u, v)+[\varphi(\lambda)-1] e, \quad(u, v) \in \bar{P}_{h, e}, \lambda \in(0,1) .
\end{aligned}
$$

Hence, $T$ is a $\varphi$-(h,e)-concave operator.

Next we show that $T: \bar{P}_{h, e} \rightarrow E \times E$ is increasing. For $(u, v) \in \bar{P}_{h, e}$, we have $(u, v)+e \in \bar{P}_{h}$. From Lemma 2.6, $\left(u+e_{1}, v+e_{2}\right) \in P_{h_{1}} \times P_{h_{2}}$. So there are $\lambda_{1}, \lambda_{2}>0$ such that

$$
u(t)+e_{1}(t) \geq \lambda_{1} h_{1}(t), \quad v(t)+e_{2}(t) \geq \lambda_{2} h_{2}(t), \quad t \in[0,1] .
$$

Therefore, $u(t) \geq \lambda_{1} h_{1}(t)-e_{1}(t) \geq-e_{1}(t) \geq-e_{1}^{*}, v(t) \geq \lambda_{2} h_{2}(t)-e_{2}(t) \geq-e_{2}(t) \geq-e_{2}^{*}$. By $\left(H_{1}\right)$ and the definitions of $A_{1}, A_{2}$, we obtain $T: \bar{P}_{h, e} \rightarrow E \times E$ is increasing. 
Now we prove that $T h \in \bar{P}_{h, e}$, so we need to prove $T h+e \in \bar{P}_{h}$. For $t \in[0,1]$,

$$
\begin{aligned}
T h(t)+e(t) & =T\left(h_{1}, h_{2}\right)(t)+e(t)=\left(A_{1} h_{1}(t), A_{2} h_{2}(t)\right)+\left(e_{1}(t), e_{2}(t)\right) \\
& =\left(A_{1} h_{1}(t)+e_{1}(t), A_{2} h_{2}(t)+e_{2}(t)\right) .
\end{aligned}
$$

We discuss $A_{1} h_{1}(t)+e_{1}(t), A_{2} h_{2}(t)+e_{2}(t)$, respectively. By Lemma 2.4 and $\left(H_{1}\right),\left(H_{3}\right)$,

$$
\begin{aligned}
A_{1} h_{1}(t)+e_{1}(t) & =\int_{0}^{1} G_{1 \alpha}(t, s) f\left(s, h_{2}(s)\right) d s \\
& \geq \int_{0}^{1} \frac{(\alpha-1) \sigma_{3} s(1-s)^{\alpha-1}}{\left(1-\sigma_{1}\right) \Gamma(\alpha)} t^{\alpha-1} f\left(s, M_{2} s^{\alpha-1}\right) d s \\
& \geq \frac{(\alpha-1) \sigma_{3}}{\left(1-\sigma_{1}\right) \Gamma(\alpha)} t^{\alpha-1} \int_{0}^{1} s(1-s)^{\alpha-1} f(s, 0) d s \\
& =\frac{(\alpha-1) \sigma_{3}}{\left(1-\sigma_{1}\right) M_{1} \Gamma(\alpha)} h_{1}(t) \int_{0}^{1} s(1-s)^{\alpha-1} f(s, 0) d s \\
A_{1} h_{1}(t)+e_{1}(t) & \leq \int_{0}^{1} \frac{(1-s)^{\alpha-1} t^{\alpha-1}}{\left(1-\sigma_{1}\right) \Gamma(\alpha)} f\left(s, M_{2}\right) d s \\
& =\frac{1}{M_{1}\left(1-\sigma_{1}\right) \Gamma(\alpha)} h_{1}(t) \int_{0}^{1}(1-s)^{\alpha-1} f\left(s, M_{2}\right) d s .
\end{aligned}
$$

From $\left(H_{1}\right),\left(H_{3}\right)$, one has $\int_{0}^{1}(1-s)^{\alpha-1} f\left(s, M_{2}\right) d s \geq \int_{0}^{1} s(1-s)^{\alpha-1} f(s, 0) d s>0$. Note that $\sigma_{3} \leq$ $\sigma_{1}<1$ and $\alpha-1 \leq 1$, we obtain

$$
\begin{aligned}
l_{1} & :=\frac{(\alpha-1) \sigma_{3}}{\left(1-\sigma_{1}\right) H_{1} \Gamma(\alpha)} \int_{0}^{1} s(1-s)^{\alpha-1} f(s, 0) d s \\
& \leq l_{2}:=\frac{1}{H_{1}\left(1-\sigma_{1}\right) \Gamma(\alpha)} \int_{0}^{1}(1-s)^{\alpha-1} f\left(s, M_{2}\right) d s,
\end{aligned}
$$

and thus $l_{1} h_{1}(t) \leq A_{1} h_{1}(t)+e_{1}(t) \leq l_{2} h_{1}(t)$. This shows $A_{1} h_{1}+e_{1} \in P_{h_{1}}$. Similarly, by using Lemma 2.4 and $\left(H_{1}\right),\left(H_{3}\right)$, we also can get $A_{2} h_{2}+e_{2} \in P_{h_{2}}$. Consequently, by Lemma 2.7,

$$
T h+e=\left(A_{1} h_{1}+e_{1}, A_{2} h_{2}+e_{2}\right) \in P_{h_{1}} \times P_{h_{2}}=\bar{P}_{h} .
$$

Finally, by using Lemma $2.5, T$ has a unique fixed point $\left(u^{*}, v^{*}\right) \in \bar{P}_{h, e}$. In addition, for any given $\left(u_{0}, v_{0}\right) \in \bar{P}_{h, e}$, the sequence

$$
\left(u_{n}, v_{n}\right)=\left(A_{1} u_{n-1}, A_{2} v_{n-1}\right), \quad n=1,2, \ldots
$$

converges to $\left(u^{*}, v^{*}\right)$ as $n \rightarrow \infty$. Therefore, system (1.1) has a unique solution $\left(u^{*}, v^{*}\right)$ in $\bar{P}_{h, e}$; taking any point $\left(u_{0}, v_{0}\right) \in \bar{P}_{h, e}$, construct the following sequences:

$$
\begin{gathered}
u_{n+1}(t)=\int_{0}^{1} G_{1 \alpha}(t, s) f\left(s, v_{n}(s)\right) d s-a \int_{0}^{1} G_{1 \alpha}(t, s) d s, \\
v_{n+1}(t)=\int_{0}^{1} G_{1 \beta}(t, s) g\left(s, u_{n}(s)\right) d s-b \int_{0}^{1} G_{1 \beta}(t, s) d s, \\
n=0,1,2, \ldots, \text { we have } u_{n+1}(t) \rightarrow u^{*}(t), v_{n+1}(t) \rightarrow v^{*}(t) \text { as } n \rightarrow \infty .
\end{gathered}
$$


Example 3.1 Consider the coupled system:

$$
\begin{cases}D^{\frac{3}{2}} v(t)+\left(\frac{1280 \sqrt[3]{140}}{3087} u(t)+\frac{1}{\Gamma\left(\frac{4}{3}\right)}\right)^{\frac{1}{5}}\left(\frac{147}{160}-\frac{3}{4} t\right)^{\frac{1}{5}} t^{\frac{1}{15}}=1, & 0<t<1, \\ D^{\frac{4}{3}} u(t)+\left(\frac{1215 \sqrt{15}}{1372} v(t)+\frac{1}{\Gamma\left(\frac{3}{2}\right)}\right)^{\frac{1}{5}}\left(\frac{98}{135}-\frac{2}{3} t\right)^{\frac{1}{5}} t^{\frac{1}{10}}=1, & 0<t<1, \\ u(0)=0, \quad u(1)=\int_{0}^{1} t^{2} u(t) d t, \quad v(0)=0, & v(1)=\int_{0}^{1} t v(t) d t .\end{cases}
$$

In this example, $\alpha=\frac{3}{2}, \beta=\frac{4}{3}, a=b=1, \varphi(t)=t^{2}, \psi(t)=t$ and

$$
\begin{aligned}
& f(t, x)=\left(\frac{1280 \sqrt[3]{140}}{3087} x+\frac{1}{\Gamma\left(\frac{4}{3}\right)}\right)^{\frac{1}{5}}\left(\frac{147}{160}-\frac{3}{4} t\right)^{\frac{1}{5}} t^{\frac{1}{15}} \\
& g(t, x)=\left(\frac{1215 \sqrt{15}}{1372} x+\frac{1}{\Gamma\left(\frac{3}{2}\right)}\right)^{\frac{1}{5}}\left(\frac{98}{135}-\frac{2}{3} t\right)^{\frac{1}{5}} t^{\frac{1}{10}}
\end{aligned}
$$

$f, g:[0,1] \times(-\infty,+\infty) \rightarrow(-\infty,+\infty)$ are continuous and increasing with respect to the second variable. After a simple computation, we have

$$
\sigma_{1}=\frac{2}{7}, \quad \sigma_{2}=\frac{3}{7}, \quad \sigma_{3}=\frac{4}{63}, \quad \sigma_{4}=\frac{9}{70} .
$$

Evidently, $\sigma_{1}, \sigma_{2} \in(0,1), \sigma_{3}, \sigma_{4}>0$. Moreover,

$$
\begin{aligned}
G_{1 \alpha}(t, s) & =G_{2 \alpha}(t, s)+G_{3 \alpha}(t, s), \quad G_{1 \beta}(t, s)=G_{2 \beta}(t, s)+G_{3 \beta}(t, s), \\
G_{2 \alpha}(t, s) & = \begin{cases}\frac{t^{\frac{1}{2}}(1-s)^{\frac{1}{2}}-(t-s)^{\frac{1}{2}}}{\Gamma\left(\frac{3}{2}\right)}, & s \leq t, \\
\frac{t^{\frac{1}{2}}(1-)^{\frac{1}{2}}}{\Gamma\left(\frac{3}{2}\right)}, & t \leq s,\end{cases} \\
G_{2 \beta}(t, s) & = \begin{cases}\frac{t^{\frac{1}{3}}(1-s)^{\frac{1}{3}}-(t-s)^{\frac{1}{3}}}{\Gamma\left(\frac{4}{3}\right)}, & s \leq t, \\
\frac{t^{\frac{1}{3}}(1-s)^{\frac{1}{3}}}{\Gamma\left(\frac{4}{3}\right)}, & t \leq s,\end{cases} \\
G_{3 \alpha}(t, s) & =\frac{t^{\frac{1}{2}}}{1-\sigma_{1}} \int_{0}^{1} t^{2} G_{2 \alpha}(t, s) d t \\
& =\frac{t^{\frac{1}{2}}}{\frac{5}{7} \Gamma\left(\frac{3}{2}\right)}\left\{\int_{0}^{s} t^{2} \cdot t^{\frac{1}{2}}(1-s)^{\frac{1}{2}} d t+\int_{s}^{1} t^{2} \cdot\left[t^{\frac{1}{2}}(1-s)^{\frac{1}{2}}-(t-s)^{\frac{1}{2}}\right] d t\right\} \\
& =\frac{2 t^{\frac{1}{2}}}{75 \Gamma\left(\frac{3}{2}\right)}\left[15(1-s)^{\frac{1}{2}}-35(1-s)^{\frac{3}{2}}+28(1-s)^{\frac{5}{2}}-8(1-s)^{\frac{7}{2}}\right]
\end{aligned}
$$

and

$$
\begin{aligned}
G_{3 \beta}(t, s) & =\frac{t^{\frac{1}{3}}}{1-\sigma_{2}} \int_{0}^{1} t G_{2 \beta}(t, s) d t \\
& =\frac{t^{\frac{1}{3}}}{\frac{4}{7} \Gamma\left(\frac{4}{3}\right)}\left\{\int_{0}^{s} t \cdot t^{\frac{1}{3}}(1-s)^{\frac{1}{3}} d t+\int_{s}^{1} t\left[t^{\frac{1}{3}}(1-s)^{\frac{1}{3}}-(t-s)^{\frac{1}{3}}\right] d t\right\} \\
& =\frac{3 t^{\frac{1}{3}}}{16 \Gamma\left(\frac{4}{3}\right)}\left[4(1-s)^{\frac{1}{3}}-7(1-s)^{\frac{4}{3}}+3(1-s)^{\frac{7}{3}}\right] .
\end{aligned}
$$


Further,

$$
\begin{aligned}
& e_{1}(t)=\int_{0}^{1} G_{1 \alpha}(t, s) d s=\frac{1}{\Gamma\left(\frac{3}{2}\right)}\left(\frac{98}{135} t^{\frac{1}{2}}-\frac{2}{3} t^{\frac{3}{2}}\right), \\
& e_{2}(t)=\int_{0}^{1} G_{1 \beta}(t, s) d s=\frac{1}{\Gamma\left(\frac{4}{3}\right)}\left(\frac{147}{160} t^{\frac{1}{3}}-\frac{3}{4} t^{\frac{4}{3}}\right), \\
& e_{1}^{*}=\max \left\{e_{1}(t): t \in[0,1]\right\}=\frac{1372}{1215 \sqrt{15} \Gamma\left(\frac{3}{2}\right)} \\
& e_{2}^{*}=\max \left\{e_{2}(t): t \in[0,1]\right\}=\frac{3087}{1280 \sqrt[3]{140} \Gamma\left(\frac{4}{3}\right)}
\end{aligned}
$$

Take $h_{1}(t)=M_{1} t^{\frac{1}{2}}, h_{2}(t)=M_{2} t^{\frac{1}{3}}$, where

$$
\begin{aligned}
& M_{1} \geq \frac{a}{\Gamma(\alpha+1)\left(1-\sigma_{1}\right)}=\frac{7}{5 \Gamma\left(\frac{5}{2}\right)}, \\
& M_{2} \geq \frac{b}{\Gamma(\beta+1)\left(1-\sigma_{2}\right)}=\frac{7}{4 \Gamma\left(\frac{7}{3}\right)} .
\end{aligned}
$$

Then

$$
\begin{aligned}
& e_{1}(t)=\frac{t^{\frac{1}{2}}}{\Gamma\left(\frac{3}{2}\right)}\left(\frac{98}{135}-\frac{2}{3} t\right) \leq \frac{98 t^{\frac{1}{2}}}{135 \Gamma\left(\frac{3}{2}\right)}<\frac{49}{45 \Gamma\left(\frac{5}{2}\right)} t^{\frac{1}{2}}<\frac{7}{5 \Gamma\left(\frac{5}{2}\right)} t^{\frac{1}{2}} \leq M_{1} t^{\frac{1}{2}}=h_{1}(t), \\
& e_{2}(t)=\frac{t^{\frac{1}{3}}}{\Gamma\left(\frac{4}{3}\right)}\left(\frac{147}{160}-\frac{3}{4} t\right) \leq \frac{147 t^{\frac{1}{3}}}{160 \Gamma\left(\frac{4}{3}\right)}<\frac{49}{40 \Gamma\left(\frac{4}{3}\right)} t^{\frac{1}{3}}<\frac{7}{4 \Gamma\left(\frac{7}{3}\right)} t^{\frac{1}{3}} \leq M_{2} t^{\frac{1}{3}}=h_{2}(t) .
\end{aligned}
$$

$g(t, 0)=\left(\frac{1}{\Gamma\left(\frac{3}{2}\right)}\right)^{\frac{1}{5}}\left(\frac{98}{135}-\frac{2}{3} t\right)^{\frac{1}{5}} t^{\frac{1}{10}} \geq 0$ with $g(t, 0) \not \equiv 0, f(t, 0)=\left(\frac{1}{\Gamma\left(\frac{4}{3}\right)}\right)^{\frac{1}{5}}\left(\frac{147}{160}-\frac{3}{4} t\right)^{\frac{1}{5}} t^{\frac{1}{15}} \geq 0$ with $f(t, 0) \not \equiv 0$. In addition,

$$
\begin{aligned}
f(t, x) & =\left(\frac{1280 \sqrt[3]{140}}{3087} x+\frac{1}{\Gamma\left(\frac{4}{3}\right)}\right)^{\frac{1}{5}}\left(\frac{147}{160}-\frac{3}{4} t\right)^{\frac{1}{5}} t^{\frac{1}{15}} \\
& =\left(\frac{1}{\Gamma\left(\frac{4}{3}\right)}\right)^{\frac{1}{5}}\left(\frac{1280 \sqrt[3]{140} \Gamma\left(\frac{4}{3}\right)}{3087} x+1\right)^{\frac{1}{5}}\left(\frac{147}{160} t^{\frac{1}{3}}-\frac{3}{4} t^{\frac{4}{3}}\right)^{\frac{1}{5}} \\
& =\left(\frac{1280 \sqrt[3]{140} \Gamma\left(\frac{4}{3}\right)}{3087} x+1\right)^{\frac{1}{5}}\left[e_{2}(t)\right]^{\frac{1}{5}} \\
& =\left(\frac{1280 \sqrt[3]{140} \Gamma\left(\frac{4}{3}\right)}{3087} x e_{2}(t)+e_{2}(t)\right)^{\frac{1}{5}}, \\
g(t, x) & =\left(\frac{1}{\Gamma\left(\frac{3}{2}\right)}\right)^{\frac{1}{5}}\left(\frac{1215 \sqrt{15} \Gamma\left(\frac{3}{2}\right)}{1372} x+1\right)^{\frac{1}{5}}\left(\frac{98}{135} t^{\frac{1}{2}}-\frac{2}{3} t^{\frac{3}{2}}\right)^{\frac{1}{5}} \\
& =\left(\frac{1215 \sqrt{15} \Gamma\left(\frac{3}{2}\right)}{1372} x+1\right)^{\frac{1}{5}}\left[e_{1}(t)\right]^{\frac{1}{5}} \\
& =\left(\frac{1215 \sqrt{15} \Gamma\left(\frac{3}{2}\right)}{1372} x e_{1}(t)+e_{1}(t)\right)^{\frac{1}{5}} .
\end{aligned}
$$


For $\lambda \in(0,1), x \in(-\infty,+\infty), y \in\left[0, e_{2}^{*}\right]$,

$$
\begin{aligned}
f( & t \lambda x+(\lambda-1) y) \\
& =\left\{\frac{1280 \sqrt[3]{140} \Gamma\left(\frac{4}{3}\right)}{3087} e_{2}(t)[\lambda x+(\lambda-1) y]+e_{2}(t)\right\}^{\frac{1}{5}} \\
& =\lambda^{\frac{1}{5}}\left\{\frac{1280 \sqrt[3]{140} \Gamma\left(\frac{4}{3}\right)}{3087} e_{2}(t)\left[x+\left(1-\frac{1}{\lambda}\right) y\right]+\frac{1}{\lambda} e_{2}(t)\right\}^{\frac{1}{5}} \\
& =\lambda^{\frac{1}{5}}\left\{\frac{1280 \sqrt[3]{140} \Gamma\left(\frac{4}{3}\right)}{3087} e_{2}(t) x+\left(1-\frac{1}{\lambda}\right) \frac{1280 \sqrt[3]{140} \Gamma\left(\frac{4}{3}\right)}{3087} e_{2}(t) y+\frac{1}{\lambda} e_{2}(t)\right\}^{\frac{1}{5}} \\
& \geq \lambda^{\frac{1}{5}}\left\{\frac{1280 \sqrt[3]{140} \Gamma\left(\frac{4}{3}\right)}{3087} e_{2}(t) x+\left(1-\frac{1}{\lambda}\right) e_{2}(t)+\frac{1}{\lambda} e_{2}(t)\right\}^{\frac{1}{5}} \\
& =\lambda^{\frac{1}{5}}\left\{\frac{1280 \sqrt[3]{140} \Gamma\left(\frac{4}{3}\right)}{3087} e_{2}(t) x+e_{2}(t)\right\}^{\frac{1}{5}} \\
& =\lambda^{\frac{1}{5}} f(t, x)=\varphi(\lambda) f(t, x),
\end{aligned}
$$

here $\varphi(\lambda)=\lambda^{\frac{1}{5}}$. By Theorem 3.1, system (3.2) has a unique nontrivial solution $\left(u^{*}, v^{*}\right)$ in $\bar{P}_{h, e}$, where

$$
\begin{aligned}
& e(t)=\left(e_{1}(t), e_{2}(t)\right)=\left(\frac{1}{\Gamma\left(\frac{3}{2}\right)}\left(\frac{98}{135} t^{\frac{1}{2}}-\frac{2}{3} t^{\frac{3}{2}}\right), \frac{1}{\Gamma\left(\frac{4}{3}\right)}\left(\frac{147}{160} t^{\frac{1}{3}}-\frac{3}{4} t^{\frac{4}{3}}\right)\right), \\
& h(t)=\left(h_{1}(t), h_{2}(t)\right)=\left(M_{1} t^{\frac{1}{2}}, M_{2} t^{\frac{1}{3}}\right), \quad t \in[0,1] .
\end{aligned}
$$

Taking any point $\left(u_{0}, v_{0}\right) \in \bar{P}_{h, e}$, construct the following sequences:

$$
\begin{gathered}
u_{n+1}(t)=\int_{0}^{1} G_{1 \alpha}(t, s) f\left(s, v_{n}(s)\right) d s-\frac{1}{\Gamma\left(\frac{3}{2}\right)}\left(\frac{98}{135} t^{\frac{1}{2}}-\frac{2}{3} t^{\frac{3}{2}}\right), \\
v_{n+1}(t)=\int_{0}^{1} G_{1 \beta}(t, s) g\left(s, u_{n}(s)\right) d s-\frac{1}{\Gamma\left(\frac{4}{3}\right)}\left(\frac{147}{160} t^{\frac{1}{3}}-\frac{3}{4} t^{\frac{4}{3}}\right), \\
n=0,1,2, \ldots, \text { we have } u_{n+1}(t) \rightarrow u^{*}(t), v_{n+1}(t) \rightarrow v^{*}(t) \text { as } n \rightarrow \infty .
\end{gathered}
$$

\section{Conclusions}

Recently, fractional coupled systems of differential equations have gained more attention in different fields of science and engineering such as physics, control systems and dynamical systems. So, for nonlinear coupled systems subject to different boundary conditions, there are many articles studying the existence or multiplicity of solutions or positive solutions. But the unique results are very rare. In this paper, we study the new coupled system of fractional differential equations (1.1). Our method is a new fixed point theorem of increasing $\varphi$ - $(h, e)$-concave operators. We present the existence and uniqueness of solutions for (1.1) dependent on two constants. Our result shows that the unique solution exists in a product set $\bar{P}_{h, e}=P_{h_{1}, e_{1}} \times P_{h_{2}, e_{2}}$ and can be approximated by making an iterative sequence for any initial point in $\bar{P}_{h, e}$. Finally, an interesting example is presented to demonstrate the main result. 
Acknowledgements

Not applicable.

\section{Funding}

The research was supported by the Youth Science Foundation of China (11201272) and Shanxi Province Science Foundation (2015011005), 131 Talents Project of Shanxi Province (2015).

\section{List of abbreviations}

Not applicable.

\section{Availability of data and materials}

Not applicable.

\section{Competing interests}

The authors declare that they have no competing interests.

\section{Authors' contributions}

The authors declare that the study was realized in collaboration with the same responsibility. All authors read and approved the final manuscript.

\section{Publisher's Note}

Springer Nature remains neutral with regard to jurisdictional claims in published maps and institutional affiliations.

\section{Received: 21 September 2017 Accepted: 15 December 2017 Published online: 04 January 2018}

\section{References}

1. Kilbas, A, Srivastava, H, Trujillo, J: In: Theory and Applications of Fractional Differential Equations. North-Holland Mathematics Studies, vol. 204. Elsevier, Amsterdam (2006)

2. Podlubny, I: Fractional Differential Equations. Academic Press, New York (1993)

3. Yang, C, Zhai, C, Zhang, L: Local uniqueness of positive solutions for a coupled system of fractional differential equations with integral boundary conditions. Adv. Differ. Equ. 2017, 282 (2017)

4. Agarwal, RP, Ahmad, B, Garout, D, Alsaedi, A: Existence results for coupled nonlinear fractional differential equations equipped with nonlocal coupled flux and multi-point boundary conditions. Chaos Solitons Fractals 102, 149-161 (2017)

5. Ahmad, B, Ntouyas, SK: A fully Hadamard type integral boundary value problem of a coupled system of fractional differential equations. Fract. Calc. Appl. Anal. 17(2), 348-360 (2014)

6. Ahmad, B, Ntouyas, SK: Existence results for a coupled system of Caputo type sequential fractional differential equations with nonlocal integral boundary conditions. Appl. Math. Comput. 266, 615-622 (2015)

7. Ahmad, B, Nieto, J: Existence results for a coupled system of nonlinear fractional differential equations with three-point boundary conditions. Comput. Math. Appl. 58, 1838-1843 (2009)

8. Ahmad, B, Nieto, JJ, Alsaedi, A, Aqlan, MH: A coupled system of Caputo-type sequential fractional differential equations with coupled (periodic/anti-periodic type) boundary conditions. Mediterr. J. Math. 6(14) 227 (2017)

9. Ahmad, B, Ntouyas, SK, Alsaedi, A: On a coupled system of fractional differential equations with coupled nonlocal and integral boundary conditions. Chaos Solitons Fractals 83, 234-241 (2016)

10. Ahmad, B, Luca, R: Existence of solutions for a sequential fractional integro-differential system with coupled integral boundary conditions. Chaos Solitons Fractals 104, 378-388 (2017)

11. Feng, M, Zhang, X, Ge, W: New existence results for higher-order nonlinear fractional differential equation with integral boundary conditions. Bound. Value Probl. 2011, Article ID 720702 (2011)

12. Hao, M, Zhai, C: Application of Schauder fixed point theorem to a coupled system of differential equations of fractional order. J. Nonlinear Sci. Appl. 7, 131-137 (2014)

13. Henderson, J, Luca, R: Positive solutions for a system of fractional differential equations with coupled integral boundary conditions. Appl. Math. Comput. 249, 182-197 (2014)

14. Liu, S, Wang, G, Zhang, L: Existence results for a coupled system of nonlinear neutral fractional differential equations. Appl. Math. Lett. 26, 1120-1124 (2013)

15. Ntouyas, SK, Obaid, M: A coupled system of fractional differential equations with nonlocal integral boundary conditions. Adv. Differ. Equ. 2012, 130 (2012)

16. Su, X: Boundary value problem for a coupled system of nonlinear fractional differential equations. Appl. Math. Lett. 22, 64-69 (2009)

17. Sun, S, Li, Q, Li, Y: Existence and uniqueness of solutions for a coupled system of multi-term nonlinear fractional differential equations. Comput. Math. Appl. 64, 3310-3320 (2012)

18. Wang, J, Xiang, H, Liu, Z: Positive solution to nonzero boundary values problem for a coupled system of nonlinear fractional differential equations. Int. J. Differ. Equ. 2010, Article ID 186928 (2010)

19. Wang, J, Zhang, Y: On the concept and existence of solutions for fractional impulsive systems with Hadamard derivatives. Appl. Math. Lett. 39, 85-90 (2015)

20. Xu, N, Liu, W: Iterative solutions for a coupled system of fractional differential-integral equations with two-point boundary conditions. Appl. Math. Comput. 244, 903-911 (2014)

21. Yang, W: Positive solutions for a coupled system of nonlinear fractional differential equations with integral boundary conditions. Comput. Math. Appl. 63, 288-297 (2012)

22. Yang, W: Positive solutions for nonlinear semipositone $q$-difference system with coupled integral boundary conditions. Appl. Math. Comput. 244, 702-725 (2014) 
23. Yuan, C, Jiang, D, O'Regan, D: Multiple positive solutions to systems of nonlinear semipositone fractional differential equations with coupled boundary conditions. Electron. J. Qual. Theory Differ. Equ. 13, 1 (2012)

24. Zhai, C, Hao, M: Multiple positive solutions to nonlinear boundary value problems of a system for fractional differential equations. Sci. World J. 2014, Article ID 817542 (2014)

25. Zhao, Y, Qin, HC: Multiple solutions for a coupled system of nonlinear fractional differential equations via variational methods. Appl. Math. Comput. 257, 417-427 (2015)

26. Amman, H: Parabolic evolution equations with nonlinear boundary conditions. In: Proc. Sympos. Pure Math. vol. 45, pp. 17-27. Amer. Math. Soc., Providence (1986)

27. Amman, H: Parabolic evolution equations and nonlinear boundary conditions. J. Differ. Equ. 72, 201-269 (1998)

28. Aronson, DG: A comparison method for stability analysis of nonlinear parabolic problems. SIAM Rev. 20, 245-264 (1978)

29. Pedersen, $M$, Lin, Z: Blow-up analysis for a system of heat equations coupled through a nonlinear boundary condition. Appl. Math. Lett. 14, 171-176 (2001)

30. Infante, G: Nonlocal boundary value problems with two nonlinear boundary conditions. Commun. Appl. Anal. 12, 279-288 (2008)

31. Infante, G, Pietramala, P: Multiple nonnegative solutions of systems with coupled nonlinear boundary conditions. Math. Methods Appl. Sci. 37, 2080-2090 (2014)

32. Deng, K: Blow-up rates for parabolic systems. Zangew Math. Phys. 47, 132-143 (1996)

33. Deng, K: Global existence and blow-up for a system of heat equations with nonlinear boundary condition. Math. Methods Appl. Sci. 18, 307-315 (1995)

34. Zhai, C, Wang, L: $\varphi$-(h,e)-concave operators and applications. J. Math. Anal. Appl. 454, 571-584 (2017)

35. Zhai, C, Ren, J: Some properties of sets, fixed point theorems in ordered product spaces and applications to a nonlinear system of fractional differential equations. Topol. Methods Nonlinear Anal. 49(2), 625-645 (2017)

\section{Submit your manuscript to a SpringerOpen ${ }^{\circ}$ journal and benefit from:}

- Convenient online submission

- Rigorous peer review

- Open access: articles freely available online

- High visibility within the field

- Retaining the copyright to your article

Submit your next manuscript at $\boldsymbol{\nabla}$ springeropen.com 